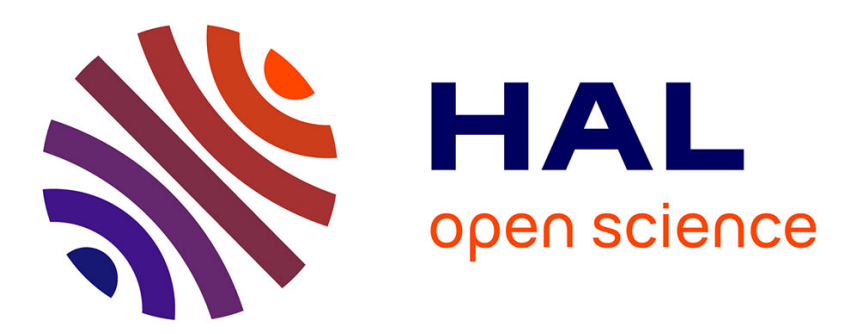

\title{
Improving Detection of Unknown Signal with Unknown Duration using an Information Criterion
}

\author{
Abigael Taylor, Olivier Rabaste
}

\section{To cite this version:}

Abigael Taylor, Olivier Rabaste. Improving Detection of Unknown Signal with Unknown Duration using an Information Criterion. 2020 IEEE Radar Conference (RadarConf20), Sep 2020, Florence, Italy. pp.1-6, 10.1109/RadarConf2043947.2020.9266522 . hal-03104152

\section{HAL Id: hal-03104152 \\ https://hal.science/hal-03104152}

Submitted on 8 Jan 2021

HAL is a multi-disciplinary open access archive for the deposit and dissemination of scientific research documents, whether they are published or not. The documents may come from teaching and research institutions in France or abroad, or from public or private research centers.
L'archive ouverte pluridisciplinaire HAL, est destinée au dépôt et à la diffusion de documents scientifiques de niveau recherche, publiés ou non, émanant des établissements d'enseignement et de recherche français ou étrangers, des laboratoires publics ou privés. 


\title{
Improving the detection of signals with noisy reference selected on a wrong-time support using an information criterion
}

\author{
Abigael Taylor and Olivier Rabaste \\ DEMR, ONERA, Université Paris-Saclay \\ F-91123 Palaiseau - France \\ Email: firstname.lastname@onera.fr
}

\begin{abstract}
Detection using a noisy reference signal is a problem that arises frequently, in particular in passive radar applications. In this paper, we go further on the misknowledge of the signal, as its time-support is supposed to be unknown - either too short or too long. We first show and illustrate that this additionnal error on the signal model deteriorates the detection performance. Two strategies to recover the time-support are proposed, the first one based on the Akaike Information Criterion, and the second one on optional additional constraints on the signal. The improvement in detection performance offered by these strategies is studied through numerical simulations.
\end{abstract}

\section{INTRODUCTION}

In classical detection theory, the optimal statistical test for detecting the presence of a signal with an unknown deterministic amplitude buried in Gaussian noise is provided by the well-known matched filter [1], [2]. This solution is in particular known to maximize the Signal-to-Noise Ratio (SNR). It however requires the knowledge of the signal to be detected. While the transmitted signal is often known in most radar applications, this is not the case when considering passive radar systems [3] or distributed active and/or passive radar systems [4].

In such settings, the most widely used strategy consists in using a reference signal, either by recording it via a dedicated reference channel [5] or by directly estimating it from the measurements. In that second case, prior information on the coding scheme of the signal can be exploited to improve the estimation, for instance in passive systems using Digital Video Broadcasting signals [6], or simply by recovering the signal from the strongest direction using a receiving antenna array [6]. We will consider in this paper that no prior knowledge on the signal is available. Besides, when the unknown signal to estimate is not a Continuous Wave but rather a train of pulses, then not only the pulse waveform is not known but also its duration must be estimated as well. Both the misknowledge of the reference signal and its duration may impact the detection performance.

This paper thus investigates the effect of using an illknown reference signal in a detection scheme. We consider a system with two channels, a reference channel providing the noisy reference signal, and a surveillance channel where the detection shall be performed. In [7], [8], the case where the reference signal is composed of the signal of interest corrupted by some additional Gaussian noise is considered, but the signal duration is assumed to be known. In that case, the solution proposed in [7] is provided by the Generalized Likelihood Ratio Test solution for an hypothesis testing problem with unknown signal amplitudes in both reference and surveillance channel, and unknown signal of interest.

In this paper, besides being noisy, the reference signal is also supposed to be selected on a wrong time-support. That is to say the measured reference signal is recorded either on a shorter or longer time duration with respect to the duration of the true signal of interest. We will first present here a study on how an incorrect time-support selection degrades the detection performance, motivating the need for estimating this duration. In that case, the classical ( decidement j'apprends bien:p) GLRT strategy that would estimate the time duration in the Maximum Likelihood sense is not pertinent since the likelihood simply increases with the duration parameter. We thus propose to resort to an information-theoretic-based method to tackle this issue, based on the well-known Akaike Information Criterion (AIC) [9]. We also consider the possibility to insert some prior knowledge on the signal waveform to estimate, namely an assumption on constant modulus of the transmitted waveform. Such an additional prior information combined with the AIC strategy enables an important performance improvement. The performance of the various stragegies considered after correcting the time-support selection will be illustrated through simulations.

This article will be organised as follows: in section II is presented the signal model and the problem statement, in section III, the impact of a wrong time-support selection is studied theoretically on simplified cases, and numerically on the considered problem; in section IV, several strategies to estimate the time-support are proposed; finally, in section $\mathrm{V}$, numerical results on the time-support estimation and its influence on the detection performance are presented.

\section{Signal MOdEL AND PROBLEM STATEMENT}

\section{A. Signal model}

Let $\mathbf{s}=\left[s_{1}, \ldots, s_{K_{0}}\right]^{T}$ be the unknown $K_{0}$-sized complex signal of interest. This signal is assumed to be received on two 
channels, and weighted by different unknown deterministic complex amplitudes $a_{1}$ and $a_{2}$ respectively. Let us assume that at the reception side, the detection processing considers received signals composed of $K$ samples to perform the detection stage.

The case where the signal duration $K_{0}$ is known, i.e. $K=$ $K_{0}$, has been studied in [4], [8]. However, in the general case, $K_{0}$ is not known and $K$ must be set either blindly or based on some rough prior knowledge of the true time duration.

Thus, let us assume here that the signal time-support $K$ of the measurement vectors $\mathbf{x}_{1}$ and $\mathbf{x}_{2}$ received on the two channels is wrongly selected, i.e. $K$ is possibly different from the true time duration $K_{0}$. Assuming that the signal will be processed with a cross-correlator that tests all the possible delays, the delay between the two channels is ignored here.

Considering the received signals are corrupted by noise, the signal model is the following:

$$
\left\{\begin{array}{l}
\mathbf{x}_{1}=a_{1} \tilde{\mathbf{s}}+\mathbf{n}_{1} \\
\mathbf{x}_{\mathbf{2}}=a_{2} \tilde{\mathbf{s}}+\mathbf{n}_{\mathbf{2}},
\end{array}\right.
$$

where for $i=1,2, \mathbf{x}_{\mathbf{i}}$ is a complex vector of size $K, \mathbf{n}_{\mathbf{i}}$ is a complex centered white Gaussian noise with variance $\sigma_{n}^{2}$ assumed known), and

$$
\left\{\begin{array}{lll}
\tilde{\mathbf{s}}=\left[\begin{array}{lll}
s_{1} & \ldots & s_{K}
\end{array}\right]^{T} & \text { if } K \leq K_{0}, \\
\tilde{\mathbf{s}}=\left[\begin{array}{lll}
s_{1} & \ldots & s_{K_{0}} 0 \ldots 0
\end{array}\right]^{T} & \text { if } K>K_{0},
\end{array}\right.
$$

depending on whether the signal support has been estimated too short or too long.

\section{B. Detection problem}

Based on Equation (1), the detection problem can be formulated as the following binary hypothesis test:

$$
\begin{aligned}
& H_{0}\left\{\begin{array}{l}
\mathbf{x}_{\mathbf{1}}=a_{1} \tilde{\mathbf{s}}+\mathbf{n}_{\mathbf{1}}, \\
\mathbf{x}_{\mathbf{2}}=\mathbf{n}_{\mathbf{2}},
\end{array}\right. \\
& H_{1}\left\{\begin{array}{l}
\mathbf{x}_{\mathbf{1}}=a_{1} \tilde{\mathbf{s}}+\mathbf{n}_{\mathbf{1}}, \\
\mathbf{x}_{\mathbf{2}}=a_{2} \tilde{\mathbf{s}}+\mathbf{n}_{\mathbf{2}} .
\end{array}\right.
\end{aligned}
$$

Without knowledge of the signal of interest time-support, the hypothesis testing problem must take into account signal $\tilde{\mathbf{s}}$ of possibly erroneous length $K$ rather than signal s of true length $K_{0}$. Based on that assumption, the log-likelihood under $H_{1}$ can be expressed as:

$\mathcal{L}_{1}=-2 K\left(\log \sigma_{n}^{2}+\log \pi\right)-\frac{1}{\sigma_{n}^{2}}\left(\left\|\mathbf{x}_{1}-a_{1} \tilde{\mathbf{s}}\right\|_{2}^{2}+\left\|\mathbf{x}_{\mathbf{2}}-a_{2} \tilde{\mathbf{s}}\right\|_{2}^{2}\right)$.

The log-likelihood under $H_{0}$ can be expressed as:

$$
\mathcal{L}_{0}=-2 K\left(\log \sigma_{n}^{2}+\log \pi\right)-\frac{1}{\sigma_{n}^{2}}\left(\left\|\mathbf{x}_{\mathbf{1}}-a_{1} \tilde{\mathbf{s}}\right\|_{2}^{2}+\left\|\mathbf{x}_{\mathbf{2}}\right\|_{2}^{2}\right) \text {. }
$$

The log-likelihood ratio test (LRT) detector is the difference between the two log-likelihoods:

$$
T_{L R T}=\mathcal{L}_{0}-\mathcal{L}_{1} \underset{H_{0}}{\stackrel{H_{1}}{\gtrless}} \eta_{L R T},
$$

with $\eta$ the threshold. However, since $a_{1}, a_{2}$ and $\mathbf{s}$ are unknown, they should be estimated. The Maximum Likelihood (ML) estimates of the amplitudes $a_{i}$ are classically provided by:

$$
\hat{a}_{i}=\frac{\tilde{\mathbf{s}}^{H} \mathbf{x}_{\mathbf{i}}}{\tilde{\mathbf{s}}^{H} \tilde{\mathbf{s}}} .
$$

Replacing the amplitudes $a_{i}$ by their estimate and removing terms that do not depend on the unknown parameters leads to the following expression for the likelihood under $H_{1}$ :

$$
\max _{a_{i}} \mathcal{L}_{1} \propto \tilde{\mathbf{s}}^{H} \frac{\mathbf{x}_{\mathbf{1}} \mathbf{x}_{\mathbf{1}}{ }^{H}+\mathbf{x}_{\mathbf{2}} \mathbf{x}_{\mathbf{2}}{ }^{H}}{\tilde{\mathbf{s}}^{H} \tilde{\mathbf{s}}} \tilde{\mathbf{s}}
$$

and the following one under $H_{0}$ :

$$
\max _{a_{i}} \mathcal{L}_{0} \propto \tilde{\mathbf{s}}^{H} \frac{\mathbf{x}_{\mathbf{1}} \mathbf{x}_{\mathbf{1}}{ }^{H}}{\tilde{\mathbf{s}}^{H} \tilde{\mathbf{s}}} \tilde{\mathbf{s}}
$$

The term $\tilde{\mathbf{s}}^{H} \frac{\mathbf{x}_{\mathbf{1}} \mathbf{x}_{\mathbf{1}}{ }^{H}+\mathbf{x}_{\mathbf{2}} \mathbf{x}_{\mathbf{2}}{ }^{H}}{\left(\tilde{\mathbf{s}}^{H} \tilde{\mathbf{s}}\right)} \tilde{\mathbf{s}}$ corresponds to a Rayleigh quotient, so that the vector $\hat{\tilde{\mathbf{s}}}$ which maximizes this quantity is the eigenvector corresponding to the largest eigenvalue of the matrix $\hat{\mathbf{R}}=\mathbf{x}_{\mathbf{1}}{ }^{H} \mathbf{x}_{\mathbf{1}}+\mathbf{x}_{\mathbf{2}}{ }^{H} \mathbf{x}_{\mathbf{2}}$.

By replacing both $a_{i}$ and $\tilde{\mathbf{s}}$ by their estimates and since $\hat{\tilde{\mathbf{s}}}^{H} \hat{\tilde{\mathbf{s}}}=1$, the likelihood under $H_{1}$ becomes

$$
\max _{a_{i}, \tilde{\mathbf{s}}} \mathcal{L}_{1} \propto \lambda_{1}
$$

where $\lambda_{1}$ is the largest eigenvalue of $\hat{\mathbf{R}}$. Similarly, the likelihood under $H_{0}$ becomes

$$
\max _{a_{i}, \tilde{\mathbf{s}}} \mathcal{L}_{0} \propto \lambda_{0}
$$

where $\lambda_{0}$ is the largest eigenvalue of $\mathbf{x}_{\mathbf{1}} \mathbf{x}_{\mathbf{1}}{ }^{H}$.

One can obtain the following Generalized LRT (GLRT):

$$
T_{G L R T}=\lambda_{1}-\lambda_{0} \underset{H_{0}}{\stackrel{H_{1}}{\gtrless}} \eta_{G L R T} .
$$

\section{IMPACT OF THE TIME-SUPPORT SELECTION ON THE DETECTION CAPACITIES - VERSION ALTERNATIVE}

The statistical distribution of test (12) is, to our knowledge, unknown (as mentionned in [7], it is already unknown without considering different time support, although the distributions of the two eigenvalues involved taken separately are known [10]). To have some insight on how a misknowledge of the true signal duration may affect the detection capacities, we propose to study what happens first for the classical Matched Filter case where the signal used for compression is the true signal, but on the wrong time support, and second for the detection test provided by (11) considering only one single channel and an unknown signal. The Matched Filter detector corresponds to the following well-known test:

$$
T_{M F}=\frac{\left|\mathbf{s}^{H} \mathbf{x}_{\mathbf{2}}\right|^{2}}{\left|\tilde{\mathbf{s}}^{H} \tilde{\mathbf{s}}\right|}
$$

Whatever the time-support length, $T_{M F}$ follows a noncentral $\chi^{2}$ distribution with 2 degrees of freedom, and a non centrality parameter $\lambda=\left|a_{2}\right|^{2} \tilde{\mathbf{s}}^{H} \tilde{\mathbf{s}}$. This means that when $K \leq K_{0}$, the detection probability will be suboptimal compared to the case $K_{0}$, while as soon as $K \geq K_{0}$, the non 


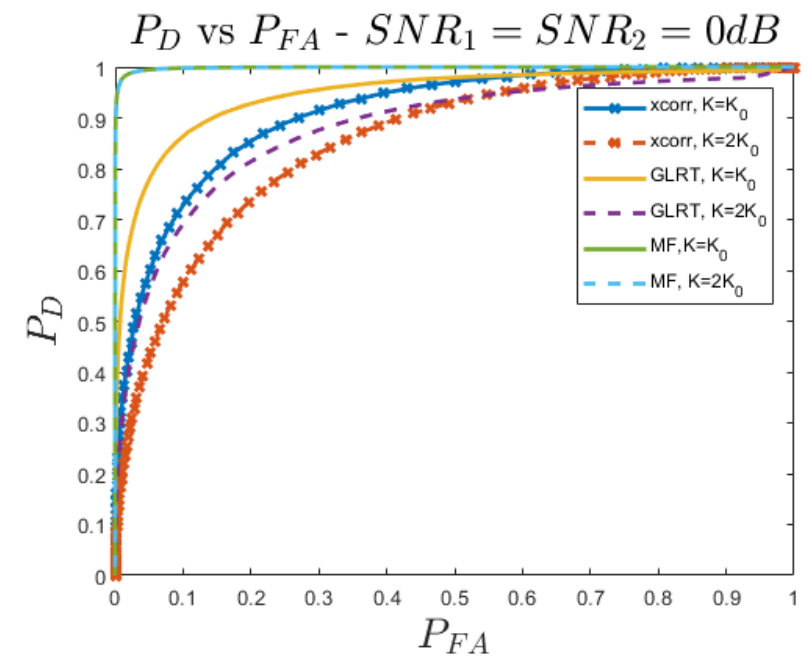

Fig. 1. Empirical probability of detection and probability of false alarm of the Matched Filter, energy detector, and the GLRT. Two signal lengths were used, $K=K_{0}$ (true length) and $K=2 K_{0}$ (dotted lines), and $S N R_{1}=$ $S N R_{2}=2 \mathrm{~dB}$.

centrality parameter remains unchanged and thus so does the detection probability. However this case is somehow biased for $K \geq K_{0}$ since it assumes a perfect knowledge of the signal and thus indirectly that the signal is composed of 0 s for length larger than $K_{0}$.

For the second considered case, provided by (11), noting that the largest eigenvector associated to $\lambda_{0}$ is simply $\tilde{\mathbf{s}}=\mathbf{x}_{1}$, it directly reduces to the energy detector, as it corresponds to the problem of detecting an unknown signal on one single channel. It is expressed as:

$$
T_{E}=\left\|\mathbf{x}_{\mathbf{2}}\right\|^{2} .
$$

In this case, the test statistics [11] is also straightforward. It follows a non-central $\chi^{2}$ distribution with $2 K$ degrees of freedom, and the same non-centrality parameter as the Matched-Filter one. When $K>K_{0}$, this non centrality parameter does not increase any more and remains constant, while the number of degrees of freedom keeps increasing, which will tend to deteriorate the detection performance.

Fig. 1 illustrates the impact of the time-support on the detection performance. $10^{5}$ Monte-Carlo simulations were run, with a post-integration gain set to $0 \mathrm{~dB}$, for a correct timesupport of $K_{0}=25$, and an overall erroneously estimated time-support of $2 K_{0}$. The empirical $P_{D}$ is represented for the Matched Filter, energy detector, and for the GLRT presented in (12), with respect to the probability of False Alarm. As expected, the Matched Filter performance is not affected by the time-support. On the contrary, for both the energy detector and the GLRT it can be noticed that the choice of the time-support does have an impact. Hence, one can expect the detection performance of the GLRT to be deteriorated as the timesupport is modified.

\section{SignAl TIME SUPPORT SELECTION USING AN INFORMATION THEORETIC CRITERION}

From these considerations, it appears that trying to correctly estimate the signal duration may improve the detection performance. In this section, we will thus first prove that this signal duration cannot be retrieved by maximizing the detection test (12) over the signal duration, and then propose two strategies that enable to estimate this time support by modifying the detection test (12).

1) Maximizing the likelihood cannot work: From (5) and (4), it can be proved that as the time-support length $K$ increases, the likelihood keeps increasing. To do so, let us set a certain length $N$ (which might exceed $K_{0}$ ). From (8), the quantity to be maximized with respect to $\tilde{\mathrm{s}}$ for a $N$-length support can be written as:

$$
\begin{array}{r}
\max _{a_{i}} \mathcal{L}_{1}(N)=-2 K\left(\sigma_{n}^{2}+\log \pi\right)-\frac{1}{\sigma_{n}^{2}}\left(\mathbf{x}_{\mathbf{1}}{ }^{H} \mathbf{x}_{\mathbf{1}}+\mathbf{x}_{\mathbf{2}}{ }^{H} \mathbf{x}_{\mathbf{2}}\right) \\
+\frac{1}{\sigma_{n}^{2}}\left(\frac{\left|\tilde{\mathbf{s}}_{\mid N}^{H} \mathbf{x}_{1 \mid N}\right|^{2}}{\tilde{\mathbf{s}}_{\mid N}^{H} \tilde{\mathbf{s}}_{\mid N}}+\frac{\left|\tilde{\mathbf{s}}_{\mid N}^{H} \mathbf{x}_{2 \mid N}\right|^{2}}{\tilde{\mathbf{s}}_{\mid N}^{H} \tilde{\mathbf{s}}_{\mid N}}\right)
\end{array}
$$

where the notation $\mathbf{v}_{\mid N}$ denotes a vector restricted to a $N$ length support. The first two terms do not depend on $N$ nor on $\tilde{\mathbf{s}}$, so that the optimization problem simply reduces to:

$$
\max _{a_{i}, \mathbf{\mathbf { s }}_{\mid N}} \mathcal{L}_{1}(N)=\max _{\tilde{\mathbf{s}}_{\mid N}} \frac{\left|\tilde{\mathbf{s}}_{\mid N}^{H} \mathbf{x}_{1 \mid N}\right|^{2}}{\tilde{\mathbf{s}}_{\mid N}^{H} \tilde{\mathbf{s}}_{\mid N}}+\frac{\left|\tilde{\mathbf{s}}_{\mid N}^{H} \mathbf{x}_{2 \mid N}\right|^{2}}{\tilde{\mathbf{s}}_{\mid N}^{H} \tilde{\mathbf{s}}_{\mid N}} .
$$

For a $N+1$ support, the optimization problem becomes:

$$
\max _{a_{i}, \tilde{\mathbf{s}}_{\mid N+1}} \mathcal{L}_{1}(N+1)=\max _{\tilde{\mathbf{s}}_{\mid N+1}} \frac{\left|\tilde{\mathbf{s}}_{\mid N+1}^{H} \mathbf{x}_{1 \mid N+1}\right|^{2}}{\tilde{\mathbf{s}}_{\mid N+1}^{H} \tilde{\mathbf{s}}_{\mid N+1}}+\frac{\left|\tilde{\mathbf{s}}_{\mid N+1}^{H} \mathbf{x}_{2 \mid N+1}\right|^{2}}{\tilde{\mathbf{s}}_{\mid N+1}^{H} \tilde{\mathbf{s}}_{\mid N+1}}
$$

It is straightforward to prove that $\max _{a_{i}, \tilde{\mathbf{s}}_{\mid N+1}} \mathcal{L}_{1}(N+1) \geq \max _{a_{i}, \tilde{\mathbf{s}}_{\mid N}} \mathcal{L}_{1}(N)$. Indeed, by noting $\tilde{\mathbf{s}}_{\mid N}^{\max }$ the signal vector maximizing Eq.(16), then setting $\tilde{\mathbf{s}}_{\mid N+1}=\left[\begin{array}{ll}\left(\tilde{\mathbf{s}}_{\mid N}^{\max }\right)^{T} & 0\end{array}\right]^{T}$ clearly already provides $\max _{a_{i}} \mathcal{L}_{1}\left(N+1, \tilde{\mathbf{s}}_{\mid N+1}\right)=\max _{a_{i}, \tilde{\mathbf{s}}_{\mid N}} \mathcal{L}_{1}(N)$. This means that the maximum likelihood is always increasing with $N$. Therefore, the correct time-support cannot be obtained by maximizing the likelihood, since the likelihood will necessarily be maximum for the largest length considered, whatever the true support. Note however that this arises because $\tilde{\mathbf{s}}_{\mid N}$ can be chosen freely.

Various strategies should thus be considered in order to correct this behavior and manage to retrieve a good time support estimation. We propose two main strategies in the following.

2) Constant Modulus strategy: The first proposed strategy consists in adding a constraint on the signal waveform. For instance, we propose to impose a constant modulus constraint on the signal of interest:

$$
\left|s_{1}\right|=\cdots=\left|s_{K_{0}}\right|,
$$

that should prevent to some extent the likelihood to irremediably increase with the length, since for sufficient SNR, 
additional samples exceeding the true signal length and composed only of noise will present a very small likelihood that should enable to decrease the overall likelihood. Thus for high SNR values, this strategy is expected to provide a good signal length estimate. For low SNR values, the estimated amplitude will be close to 0 so that the behavior of the estimator provided by this strategy should be similar to the classical GLRT, and keep increasing. Note that this behavior will be mathematically explained in a subsequent paper, but the mathematical derivation is too tedious to be inserted here. In the following, this constant modulus constraint will be noted as $\mathrm{CM}$.

Let us denote by $\mathcal{C}$ the set of $\mathrm{CM}$ signals, restricted without loss of generality to signals with modulus equal to 1 . Then the likelihood should be optimized under both hypotheses with the additional constraint that $\mathbf{s} \in \mathcal{C}$. Under $H_{0}$, considering again Eq.(9), it can be easily shown that the optimal solution to the problem

$$
\max _{a_{i}, \mathbf{s} \in \mathcal{C}} \mathcal{L}_{0} \propto \max _{\mathbf{s} \in \mathcal{C}} \tilde{\mathbf{s}}^{H} \frac{\mathbf{x}_{\mathbf{1}} \mathbf{x}_{\mathbf{1}}{ }^{H}}{\tilde{\mathbf{s}}^{H} \tilde{\mathbf{s}}} \tilde{\mathbf{s}}
$$

is provided by $\hat{s}_{i}=e^{j \varphi_{i}}$ where $\varphi_{i}$ is the phase of the $i^{\text {th }}$ entry of $\mathbf{x}_{1}$. This optimization is thus straightforward.

The optimization under $H_{1}$ with the constraint $\mathbf{s} \in \mathcal{C}$ is not as simple, and should normally require the use of an optimization algorithm, which would be very costly. However recall that the optimal solution of $\max _{a_{i}, \mathbf{s} \in \mathcal{C}} \mathcal{L}_{1}$ is provided by $\lambda_{1}$ the maximum eigenvalue of matrix $\hat{\mathbf{R}}$. We propose here to consider the vector defined by entries $\hat{s}_{i}=e^{j \theta_{i}}$ where $\theta_{i}$ is the phase of the $i^{\text {th }}$ entry of the eigenvector associated to $\lambda_{1}$. This vector is not the optimal solution of the constrained optimization problem, but happens to be almost optimal, and presents the advantage of being easily computed. We will thus use this suboptimal solution in the following.

3) AIC-based strategy: The second strategy proposed in this paper in order to estimate the time-support consists in using the Akaike Information Criterion (AIC). In radar applications, the AIC is often used as a mean to choose the number of targets/signals, see e.g. [12] [13]. The AIC adds a penalty to the log-likelihood of the data, to avoid an overfitting of the data, which will be traduced here into avoiding to overestimate the signal length. It is expressed as:

$$
A I C(p)=-2 \hat{\mathcal{L}}+2 p
$$

where $p$ is the number of parameters of the model, and $\hat{\mathcal{L}}$ is the maximum value of the log-likelihood. If applied to the likelihood as expressed in Eq. (10) or Eq. (11), $p=2 K$ since the signal waveform is represented by $K$ real values and $K$ imaginary values (or $K$ modulus and $K$ phases). If applied to the likelihood with the additionnal constant modulus constraint, however, $p=K$ since then only $k$ phase values can be freely chosen.

With this criterion, the time support estimate is thus given by:

$$
\begin{aligned}
& \hat{K}_{A I C}=\arg \min _{K}[-2 \hat{\mathcal{L}}+4 K] \text { if no constraint } \\
& \hat{K}_{A I C}=\arg \min _{K}[-2 \hat{\mathcal{L}}+2 K] \text { if CM constraint }
\end{aligned}
$$

It is possible to theoretically show that this strategy will tend to provide signal length estimates close to the minimum tested length at low SNR (contrary to the classical GLRT that will always provide the maximum tested length), while converging to the true signal length for high SNR values. The proof of this behavior will be provided in a subsequent paper and will be observed on simulations in the following.

It can be noted that if these strategies were applied in a Maximum Likelihood sense, the signal length estimation strategy should be applied to each hypothesis $H_{0}$ and $H_{1}$ separately, so that the resulting estimated length may differ, accordingly to the tested hypothesis. This means that both data sets $\mathbf{x}_{1}$ and $\mathbf{x}_{2}$ will be used under $H_{1}$, which seems appropriate, since then the length would be estimated using all the available information. Under $H_{0}$, only the reference data $\mathbf{x}_{1}$ would be used. However, it appeared that considering only the reference dataset $\mathbf{x}_{1}$ for the length estimation even under $H_{1}$ was more efficient for the detection performance, so we also considered this alternative.

Mixing all the different possibilities led to the 7 strategies summarized below:

- Classical GLRT

- GLRT + CM, $K_{0}$ estimated on $\mathrm{x}_{1}$ only

- GLRT + CM, $K_{0}$ estimated on $\mathbf{x}_{1}$ under $H_{0}$, and on $\mathbf{x}_{1}$ and $\mathbf{x}_{2}$ under $H_{1}$

- GLRT + AIC, $K_{0}$ estimated on $\mathbf{x}_{1}$ only

- GLRT + AIC, $K_{0}$ estimated on $\mathbf{x}_{1}$ under $H_{0}$, and on $\mathbf{x}_{\mathbf{1}}$ and $\mathbf{x}_{2}$ under $H_{1}$

- GLRT + CM + AIC, $K_{0}$ estimated on $\mathbf{x}_{1}$ only

- GLRT + CM + AIC, $K_{0}$ estimated on $\mathbf{x}_{1}$ under $H_{0}$, and on $\mathbf{x}_{1}$ and $\mathbf{x}_{2}$ under $H_{1}$

\section{NuMERICAL RESULTS}

Simulation framework: In the following, we will set $K_{0}=50$. The tested lengths will be chosen between 25 and 100, i.e. $K_{0} / 2$ and $2 K_{0}$ respectively. In the following, the SNR of the reference and surveillance path will be denoted $S N R_{1}$ and $S N R_{2}$ respectively. For $P_{F A}$ calculations, $10^{5}$ Monte-Carlo simulations were run. These simulations were used to evaluate the thresholds to have a $10^{-3}$ false alarm rate. These estimated thresholds were then used to determine $P_{D}$ over $10^{3}$ MonteCarlo simulations. To calculate the $P_{D}$ and $P_{F A}, S N R_{1}$ was set to a given value : either $10 \mathrm{~dB}, 22 \mathrm{~dB}$ or $30 \mathrm{~dB}$. The $P_{D}$ was then calculated for various values of $S N R_{2}$, taken between $5 \mathrm{~dB}$ and $30 \mathrm{~dB}$. It should be noted that the given $\mathrm{SNR}$ values correspond to a post-compression SNR, i.e. it is defined as $a_{i}^{2}\|\mathbf{s}\|^{2} / \sigma^{2}$.

Preliminary remark: From observations on numerical simulations, we decided to discard all the strategies involving an estimation of $K_{0}$ under both data sets. Indeed, apart from high SNR on both channels configurations, they all led to poor 


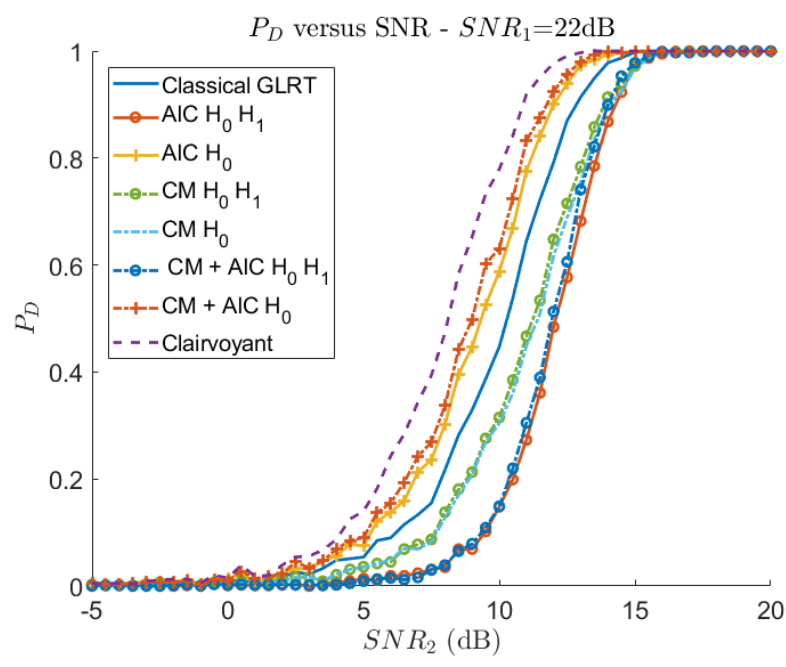

Fig. 2. Probability of detection vs $S N R_{2}$, for $S N R_{1}=22 \mathrm{~dB}$. All the strategies mentionned in section IV are tested. The curves plotted with a 'o' marker correspond to the strategies for which $K_{0}$ is estimated on both data sets, which will not be considered afterwards. The ones involving the AIC have a + marker, and the ones using a constant modulus (denoted 'CM' in the legend) constraint are plotted with a '-.' linestyle. As a comparison, the detector using the true signal (called clairvoyant), and the true signal length is plotted.

detection probability $P_{D}$, even though the estimated length were comparable to the ones obtained with the other strategies. This can be seen from Figure 2, where the $P_{D}$ obtained with these methods are worse than the classical GLRT $P_{D}$, unlike the methods using the reference channel only to estimate $K_{0}$.

Signal length estimation: As only strategies using $\mathbf{x}_{1}$ to estimate $K_{0}$ are considered, the only SNR impacting the signal length estimation is $S N R_{1}$. Figure 3 shows the mean and standard deviation of $\hat{K}_{0}$ estimated under $H_{0}$ as a function of $S N R_{1}$, calculated over 1000 monte-carlo simulations, for these strategies. As expected, the estimation with the classical GLRT always leads to the maximum tested length (in this case, $\left.\hat{K}_{0}=100\right)$. The strategy which exploits a constant modulus constraint, but not the AIC, behaves similarly to the classical GLRT for $S N R_{1}$ values below $17 \mathrm{~dB}$. Above this value, it converges towards the true $K_{0}$ value, but with notably high standard deviation values at first, and with low standard deviation values for $S N R_{1}>26 \mathrm{~dB}$. Strategies that involve the AIC start with underestimated values of the signal length. The one using both the AIC and the constant modulus constraint converges towards the true value for $S N R_{1}>15 \mathrm{~dB}$. It can be noted that the standard deviation is quite important until $S N R_{1}$ reaches about $20 \mathrm{~dB}$. The strategy using the AIC only converges a little bit later, for a $S N R_{1}$ of around $20 \mathrm{~dB}$. This strategy standard deviation is much lower that the previous one, appart for the transitionary phase, $10 \mathrm{~dB}<S N R_{1}<20 \mathrm{~dB}$.

Figure 4 presents the detection probability $P_{D}$ as a function of $S N R_{2}$ curves for $S N R_{1}=25 \mathrm{~dB}$. It can be noted that the classical GLRT gives the worst $P_{D}$, whereas all the proposed

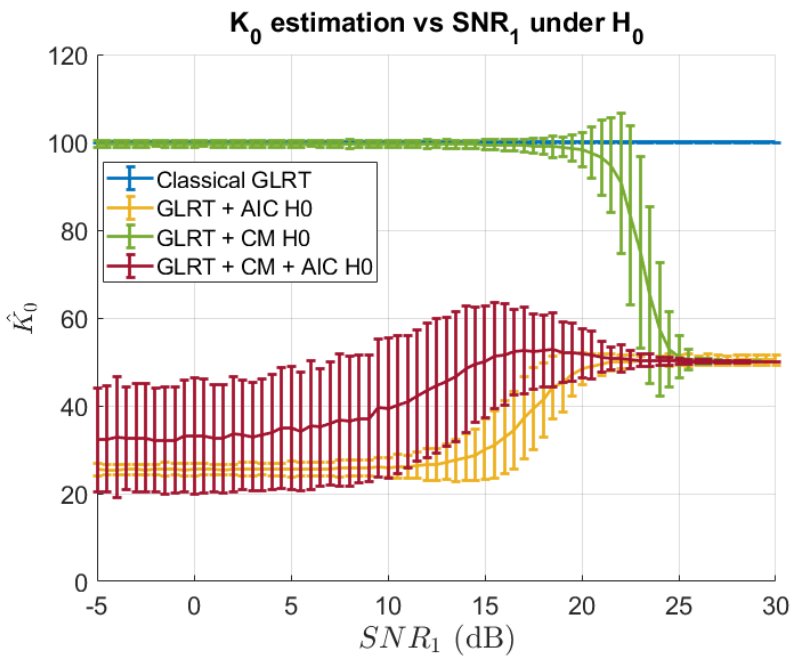

Fig. 3. Signal length estimation using different strategies AIC as a function of $S N R_{1}$. The error bars represent the estimates standard deviation.

strategies are quite close to one another, and approaches the best $P_{D}$ curve possible, obtained using both the true length and the true signal. This behavior can be explained by the fact that when $S N R_{1}=25 \mathrm{~dB}$, the signal length is correctly estimated by all proposed strategies. The $P_{D}$ obtained with the strategy using the AIC is slightly below the two others.

Figure 5 presents the detection probability as a function of $S N R_{2}$ curves for $S N R_{1}=15 \mathrm{~dB}$. Unlike in the precedent case, one strategy seems ahead of the others: using the constant modulus constraint and the AIC on the reference data only. The other ones give results close to the classical GLRT, or even worse. This is in accordance with observations made on the signal length estimation, since for this $S N R_{1}$ level the strategy using both the constant modulus constraint and the AIC is the most efficient one.

Finally, Figure 6 presents the detection probability as a function of $S N R_{2}$ curves for $S N R_{1}=10 \mathrm{~dB}$. The strategy using the constant modulus constraint and the AIC still gives better results than the classical GLRT. However, for this SNR, the other ones give clearly worse $P_{D}$ curves than the GLRT. Again this is in accordance with observations made on the signal length estimation.

\section{CONCLUSION}

In this paper, a detection problem with an unknown reference signal, and an ill-estimated signal length was considered. Various methods to estimate the reference signal time support were proposed, using the Akaike Criterion Information, a constant modulus constraint on the signal, or both. Numerical simulations showed that the performance of these strategies depend on the signal to noise ratio on the reference signal. One of the proposed strategies presents better probability of detection that the classical GLRT, the other ones being more impacted when the SNR of the reference channel decreases. Based on these results, the best strategy is the one using both the AIC and the constant modulus constraint on the signal, 


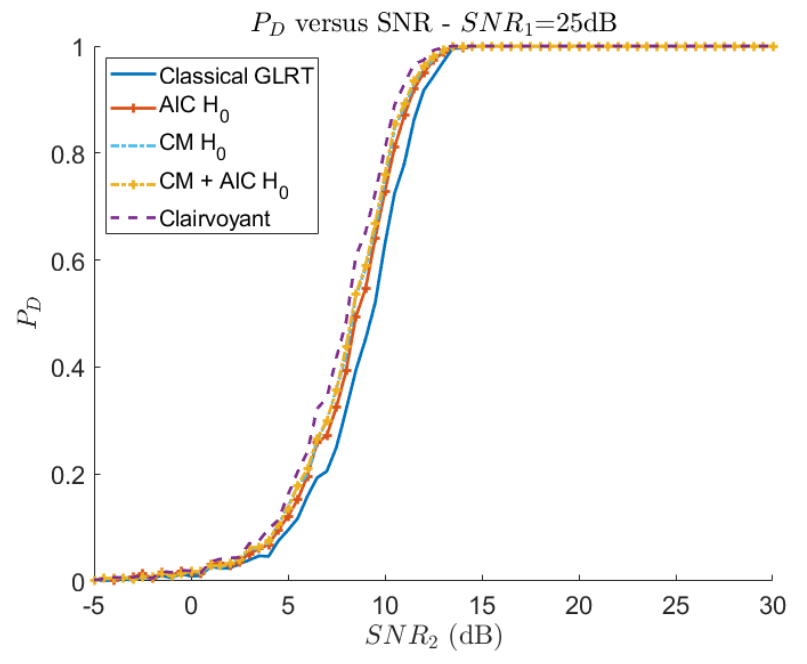

Fig. 4. $P_{d}$ vs $S N R_{2}$, in $\mathrm{dB}, S N R_{1}=25 \mathrm{~dB}$. Various strategies are tested. The ones involving the AIC have a + marker, and the ones using a constant modulus (denoted 'CM' in the legend) constraint are plotted with a '-.' linestyle. As a comparison, the detector using the true signal (called clairvoyant), and the true signal length is plotted.

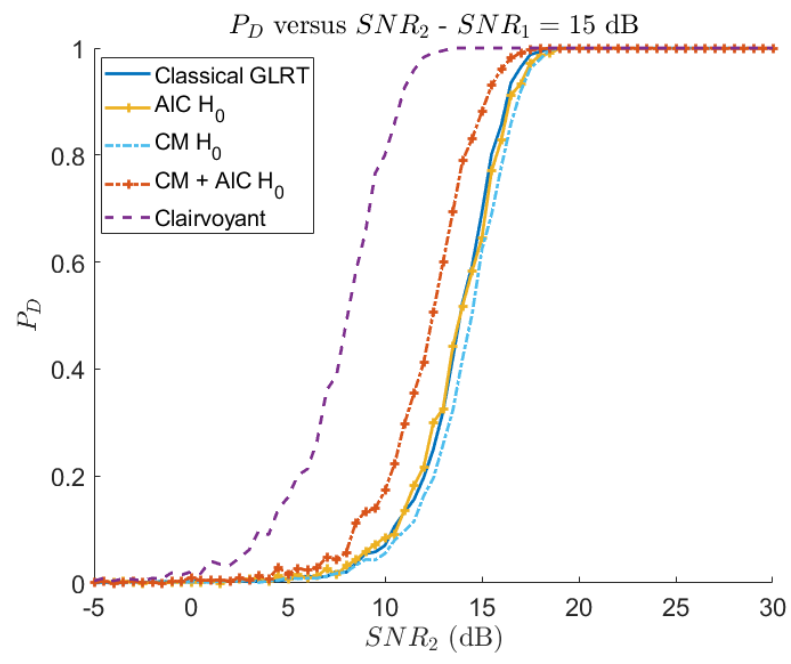

Fig. 5. $P_{d}$ vs $S N R_{2}$, in $\mathrm{dB}, S N R_{1}=15 \mathrm{~dB}$. Various strategies are tested. The ones involving the AIC have a + marker, and the ones using a constant modulus (denoted 'CM' in the legend) constraint are plotted with a '-.' linestyle. As a comparison, the detector using the true signal (called clairvoyant), and the true signal length is plotted.

which improved the probability of detection, even for lower signal to noise ratio values. Further work should be carried out on the statistics of the proposed tests, and on the behaviors of the length estimate at low or high signal to noise ratio.

\section{REFERENCES}

[1] S.M. Kay. Fundamentals of Statistical Signal Processing. Detection theory. Prentice Hall, 1998.

[2] L. Scharf and S. Kraut. Geometries, invariances, and SNR interpretations of matched and adaptive subspace detectors. Traitement du Signal, 15(6):527-534, 1998.

[3] B.D. Nordwall. "Silent sentry" A new type of Radar. Aviat. Week Space Technol., pages 70-73, 1998

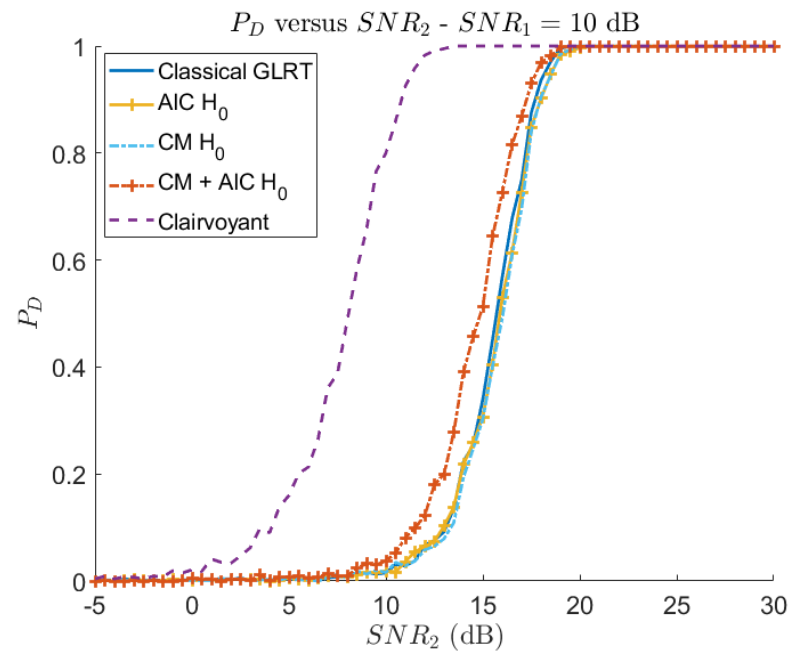

Fig. 6. $P_{d}$ vs $S N R_{2}$, in $\mathrm{dB}, S N R_{1}=10 \mathrm{~dB}$. Various strategies are tested. The ones involving the AIC have a + marker, and the ones using a constant modulus (denoted 'CM' in the legend) constraint are plotted with a '-.' linestyle. As a comparison, the detector using the true signal (called clarivoyant), and the true signal length is plotted.

[4] D.E. Hack, L.K. Patton, and B. Himed. A unified detection framework for distributed active and passive RF sensing. In 2013 Asilomar Conference on Signals, Systems and Computers, pages 449-453, 2013.

[5] F. Colone, D.W. O'Hagan, P. Lombardo, and C.J. Baker. A Multistage Processing Algorithm for Disturbance Removal and Target Detection in Passive Bistatic Radar. IEEE Transactions on aerospace and electronic systems, 45(2):698-722, 2009.

[6] D. Poullin. Passive Detection using digital broadcasters (DAB, DVB) with COFDM modulation. IEE Proc. Radar Sonar Navig., 152(3):143152, 2005.

[7] D.E. Hack, L.K. Patton, B. Himed, and M.A. Saville. Detection in Passive MIMO Radar Networks. IEEE Transactions on Signal Processing, 62(11):2999-3012, 2014.

[8] G. Cui, J. Liu, H. Li, and B. Himed. Signal detection with noisy reference for passive sensing. Signal Processing, 108:389-399, 2015.

[9] H. Akaike. Information theory and an extension of the maximum likelihood principle. In Proc. 2nd Int. Symp. Inf. Theo., pages 267281, 1973.

[10] J. Sala-Alvarez, G. Vézquez-Vilar, R. López-Valcarce, S. Sedighi, and A. Taherpour. Multiantenna GLR Detection of Rank-One Signals With Known Power Spectral Shape Under Spatially Uncorrelated Noise. IEEE Transactions on Signal Processingl, 64(23):6269 - 6283, 2016.

[11] J.I. Marcum. A Statistical Theory of Target Detection by Pulsed Radar. RAND Research Memo. RM-754, 1947.

[12] M. Wax and T. Kailath. Detection of Signals by Information Theoretic Criteria. IEEE Transactions on Acoustics, Speech and Signal Processing, ASSP-33(2), 1985.

[13] E. Fishler, M. Grosmann, and H. Messer. Detection of Signals by Information Theoretic Criteria: General Asymptotic Performance Analysis. IEEE Transactions on Signal Processing, 50(5), 2002. 